\title{
Simulations of Relativistic Jet Formation in Compact Radio Sources
}

\author{
David L. Meier \\ Jet Propulsion Laboratory, California Institute of Technology, \\ Pasadena, CA 91109, USA
}

\begin{abstract}
I review recent numerical and analytic work on the magnetohydrodynamic (MHD) model of jet formation in active galactic nuclei, with an emphasis on producing the highly relativistic outflows and high radio luminosities observed in the most powerful sources.
\end{abstract}

Ground and space very long baseline interferometry imaging observations have shown that collimated, relativistic flow at speeds up to a Lorentz factor $\Gamma=10(0.995 c)$ and greater occur in radio galaxies and quasars (Vermeulen 1996; Tingay et al. 2001). Such high jet speeds are thought to be evidence that the they are accelerated and collimated from deep within the potential well of an accreting supermassive black hole $\left(M=10^{6-10} M_{\odot}\right.$ in size $)$ at the center of the active galaxy. The total jet power for a given black hole mass can be as high as $L_{j e t} \approx 10^{45.5} \mathrm{erg} \mathrm{s}^{-1} m_{9}^{1.12}$, where $m_{9}=M / 10^{9} M_{\odot}$ (Meier 2001c).

A promising model for producing and collimating relativistic jets is the magnetohydrodynamic (MHD) acceleration model (Lovelace 1976; Blandford 1976). This model may be able to account for jet collimation, acceleration to relativistic velocities, and the magnetic fields associated with detected synchrotron emission, all while operating within the accretion paradigm for active galaxies. Recent results on numerical simulations of jet production by magnetized accretion disks also have been reviewed by Meier \& Koide (2000), Meier (2001a; 2001b), and Meier et al. (2001). These simulations are:

Time-dependent, non-relativistic, strong field, thick disk (Uchida et al. 2000). A strong poloidal magnetic field (Alfvén velocity $v_{A} \sim v_{e s c}$, the escape speed) and a differentially rotating thick disk winds up the field into a toroidal component $\left(B_{\phi}\right)$, whose vertical pressure accelerates plasma upward, and hoop stress pinches and collimates the outflow into a narrow jet with a speed of order the escape velocity from the inner edge of the disk.

Steady-state, non-relativistic, weak field, thin disk (Ouyed \& Pudritz 1997; Krasnopolsky et al. 1999). In these the disk, assumed to be infinitely thin and in permanent Keplerian rotation, ejects a slow wind along the magnetic field lines embedded in it. The wind rotation slows, bending the field lines backward and producing a slowly-collimating outflow, with terminal velocity again of order the escape speed.

Time-dependent, fully relativistic, weak field, thick disk (Koide et al. 2000). General relativistic simulations of black holes also produce jets with speeds near $v_{e s c}$. For the Schwarzschild case $v_{j e t} \sim 0.4 c$, driven by the rotation of the accretion disk. In the Kerr case $\Gamma_{j e t} \lesssim 2.7$, and the jet, formed inside the ergosphere $\left(R<2 G M / c^{2}\right)$, is driven by the rotation of the black hole itself. 
Time-dependent, non-relativistic, strong field, thin disk (Meier et al. 1997; Ustyugova et al. 2000). Similar to the steady-state ones above, these choose a much stronger magnetic field $\left(v_{A}>>v_{e s c}\right)$. In contrast to the broad wind, a highly-collimated jet develops close to the central black hole. Because $v_{A}$ can be highly relativistic for low density plasmas, and because $v_{j e t} \sim v_{A}$, the strong field case can produce jets with $\Gamma>>1$.

For a jet produced by a rotating accretion disk around a black hole, the total theoretical MHD power is given by (Livio et al. 1999 ; Meier 2001c) $L_{j e t}=$ $(H / R)^{2} B_{\text {disk }}^{2} R^{4} \Omega^{2} / 32 c$, where $B_{\text {disk }}$ is the toroidal field in the disk, $H$ is the disk scale height, $R$ is the radius where the jet is produced, and $\Omega$ is the angular velocity of the rotating field. The factor of $(H / R) B_{d i s k}$ represents the poloidal magnetic field that accelerates the jet. For standard, Shakura \& Sunyaev (1973) disks, $H<<R$, resulting in a weak poloidal field and, therefore, a weak jet of only $\sim 10^{42-43} \mathrm{erg} \mathrm{s}^{-1} m_{9}^{0.9}$, which is 2-3 orders of magnitude smaller than the maximum jet powers observed. A stronger poloidal field can be generated by a thick disk, such as an advection-dominated accretion flow (ADAF; Narayan \& Yi 1995). But because they are partially pressure-supported, ADAFs rotate at a rate well below Keplerian, again resulting in a rather weak total power of $\lesssim 10^{44} \mathrm{erg} \mathrm{s}^{-1} m_{9}$ for a Schwarzschild hole. However, if the ADAF is flowing into a rapidly-rotating Kerr hole, then it will have both a strong field and rapid rotation, yielding a total power of $z 10^{45} \mathrm{erg} \mathrm{s}^{-1} \mathrm{~m}_{9}$.

So while even thin disks around Schwarzschild holes can produce weak radio jets, only ADAFs around Kerr holes can produce the most powerful jets. This lends support to the idea that Schwarzschild holes are radio quiet, while Kerr holes produce radio loud galaxies and quasars (Wilson \& Colbert 1995).

Acknowledgments. This research was carried out at the Jet Propulsion Laboratory, California Institute of Technology, under contract to NASA.

\section{References}

Blandford, R.D. 1976, MNRAS, 176, 465.

Koide, S., Meier, D., Shibata, K., \& Kudoh 2000, ApJ, 536, 668.

Krasnopolsky, R., Li, Z-Y, \& Blandford, R. 1999, ApJ, 526, 631.

Livio, M. Ogilvie, G. I., \& Pringle, J. E. 1999, ApJ, 512, 100.

Lovelace, R.V.E. 1976, Nature, 262, 649.

Meier, D. et al.. 1997, Nature, 388, 350.

Meier, D.L. 2001a, in The 3rd Microquasar Workshop, Kluwer, in press.

Meier, D.L. 2001b, in Particles and Fields in Radio Galaxies, ASP, in press.

Meier, D.L. 2001c, ApJ Letters, in press.

Meier, D.L. \& Koide, S. 2000, in The First VSOP Symposium, ISAS, 31.

Meier, D.L., Koide, S., \& Uchida, Y. 2001, Science, January, in press.

Narayan, R. \& Yi, I. 1995, ApJ, 452, 710.

Ouyed, R. \& Pudritz, R. 1997, ApJ, 482, 712.

Shakura, N. I. \& Sunyaev, R. A. 1973, Astron. Astrophys. 24, 337.

Tingay, S.J. et al. 2001, preprint.

Uchida, Y. et al.. 2000, in IAU Symp. 195, 213.

Ustyugova, G.V. et al.. 2000, ApJ, 541, L21.

Vermeulen, R.C. 1996, in IAU Symp. 175, 57.

Wilson, A.S. \& Colbert, E.J.M. 1995, ApJ, 438, 62. 